\title{
Wege zur Beurteilung der Umweltverträglichkeit biozider Wirkstoffe
}

\author{
Walter Giger · Daniel Bürgi · Michael Burkhardt $\cdot$ Leo Morf
}

Erhalten: 14. Dezember 2008/Akzeptiert: 15. Dezember 2008/Online veröffentlicht: 17. Januar 2009

(C) Springer-Verlag 2009

\section{Problemstellung}

Biozide sind biologisch aktive Substanzen und können somit eine potenzielle Gefährdung für Umwelt und Mensch

Teil I: Daniel Bürgi · Lars Knechtenhofer · Isabel Meier · Walter Giger (2008) Priorisierung von bioziden Wirkstoffen aufgrund der potenziellen Gefährdung schweizerischer Oberflächengewässer. Umweltwiss Schadst Forsch, DOI: 10.1007/s12302-008-0032-2

Teil II: Andreas M. Buser · Leo S. Morf (2008) Stoffflussanalyse von quartären Ammoniumverbindungen für die Schweiz. Modellierung des Verbrauchs in bioziden Anwendungen und der Emissionen in die Umwelt. Umweltwiss Schadst Forsch, DOI: 10.1007/s12302-008-0025-1

Teil III: Michael Burkhardt · Marion Junghans · Steffen Zuleeg · Ute Schoknecht · Xolelwa Lamani - Kai Bester - Roger Vonbank · Hans Simmler · Markus Boller (2008) Biozide in Gebäudefassaden - ökotoxikologische Effekte, Auswaschung und Belastungsabschätzung für $\mathrm{Ge}-$ wässer. Umweltwiss Schadst Forsch, DOI: 10.1007/s12302-008-0033-1

Verantwortliche Herausgeber: Walter Giger · Daniel Bürgi · Michael Burkhardt $\cdot$ Leo Morf

\section{W. Giger $(\bowtie)$}

GRC, Giger Research Consulting,

Im oberen Boden 128, 8049 Zürich, Schweiz

E-Mail: giger@eawag.ch

D. Bürgi

FRIEDLIPARTNER AG,

Baumackerstrasse, 8050 Zürich, Schweiz

E-Mail: Daniel.Buergi@friedlipartner.ch

M. Burkhardt

Eawag - Das Wasserforschungs-Institut des ETH-Bereichs,

Abteilung Siedlungswasserwirtschaft,

Überlandstrasse 133, 8600 Dübendorf, Schweiz

E-Mail: michael.burkhardt@eawag.ch

L.S. Morf

GEO Partner AG Ressourcenmanagement,

Baumackerstrasse 24, 8050 Zürich, Schweiz

E-Mail: morf@geopartner.ch darstellen. Daraus resultiert die Notwendigkeit eines Risikomanagements. Für alle Produktebereiche wird das Vorgehen in der europäischen Biozidprodukte-Richtlinie (BPRL, 98/8/EG) und in der schweizerischen Biozidprodukteverordnung (VBP) geregelt. In diesen Regelwerken sind die Biozidprodukte in vier Hauptgruppen eingeteilt: (1) Desinfektionsmittel, (2) Schutzmittel, (3) Schädlingsbekämpfung und (4) sonstige Biozidprodukte. Innerhalb dieser Hauptgruppen werden insgesamt 23 Produktarten unterschieden. Die als Biozide eingesetzten, mehreren hundert Wirkstoffe wurden innerhalb der EU identifiziert, notifiziert und in einer Liste zusammengestellt. Die Registrierung und Zulassung der Biozidprodukte wird über die Beurteilung der darin enthaltenen Wirkstoffe erfolgen, für die von den Herstellerfirmen Grundlagendaten zur Verfügung gestellt werden müssen. Die Bereitstellung der Grundlagendaten und die Beurteilung durch die Behörde ist sowohl zeitlich als auch finanziell limitiert. Für die Prüfung und Bewertung der Wirkstoffe sollten daher die richtigen Prioritäten festgelegt werden.

Bei zahlreichen der eingesetzten Biozidprodukte ist eine relevante Emission in das aquatische System zu erwarten. Dies ist insbesondere der Fall für Biozide, die direkt in wässriger Umgebung eingesetzt werden, wie z.B. Desinfektionsmittel für Schwimmbäder, Konservierungsstoffe für Detergenzien (Wasch- und Reinigungsmittel, Körperpflegemittel), Biozide für Kühlwassersysteme oder AntifoulingProdukte. Emissionen in das aquatische System treten indirekt aber auch bei vielen anderen Anwendungen auf, wie z. B. bei den Bioziden für die menschliche Hygiene, Schutzmitteln für Materialien, Desinfektionsmitteln für Oberflächen im privaten und öffentlichen Bereich etc.

Biozide kommen aufgrund der beschriebenen Anwendungen und Emissionen als Mikroverunreinigungen in $\mathrm{Ab}-$ wasser und Gewässern vor. Die Europäische Biozidprodukte-Richtlinie und die Schweizer Biozidprodukteverordnung 
sehen daher eine Beurteilung der Umweltverträglichkeit der Wirkstoffe vor. Für die Bewertung der Biozide stellt sich aus der Sicht eines vorsorgeorientierten Verbraucher- und Umweltschutzes aber das Problem, wie eine Priorisierung der einzelnen bereits verwendeten oder der zukünftigen Wirkstoffe durchgeführt werden und dabei die neuesten Erkenntnisse zu Mikroverunreinigungen berücksichtigt werden können. Im Zusammenhang mit den genannten Regelwerken sind zunächst aber drei zentrale Schwachpunkte der Bewertungsverfahren zu nennen:

1. Die für die einzelnen Produktarten definierten Emissionsszenarien sind in einigen Fällen unvollständig hinsichtlich möglicher Eintragspfade in die Umwelt.

2. Die Beurteilung basiert auf der isolierten Bewertung von einer Substanz in einer Anwendungsart. Ein Biozid wird jedoch meistens in mehreren unterschiedlichen Produktarten eingesetzt. Die geltenden Regelungen sehen keine Gesamtbetrachtung über die unterschiedlichen Anwendungen in allen Produktarten vor.

3. Es ist keine vergleichende Betrachtung der unterschiedlichen Wirkstoffe vorgesehen.

\section{Ansätze und Methoden der Beitragsserie ,Umweltverträglichkeit biozider Wirkstoffe'}

Aus den genannten Defiziten in dem Zulassungsverfahren wurde die Umweltverträglichkeit der Biozide von Bürgi et al. (Teil I dieser Beitragsserie) erstmals in einem übergreifenden und vergleichenden Sinne beurteilt. Ergänzend zum Ansatz in den bestehenden Regelungen wird von der Gesamtexposition einer Substanz aufgrund der Anwendung in allen Produktarten ausgegangen. Basierend auf den Verbrauchsangaben, Emissionsabschätzungen und den ökotoxikologischen und chemisch-physikalischen Stoffdaten wurden Prioritäten für weitere Untersuchungen, wie Gefährdungsabschätzungen, Expositionsabschätzungen, Risikobewertungen etc. vorgeschlagen. Außerdem sollten verstärkt Modellabschätzungen und Lebenszyklus-Analysen, wie in der hier vorgestellten Studie von Buser et al. (Teil II dieser Beitragsserie), eingesetzt werden, um Biozide breiter zu evaluieren. Mittels Fehlerbetrachtungen wurden die Datenunsicherheiten geschätzt sowie die Informationslücken zusammen mit der betroffenen chemischen Industrie diskutiert und Vorschläge für ein zukünftiges Vorgehen für Biozide ausgearbeitet. Die Teile I und II wurden in enger Zusammenarbeit durchgeführt mit dem Ziel, durch Literaturrecherchen, Interviews sowie mittels einer Priorisierungsmethodik und Stoffflussanalyse abzuschätzen, welche Biozide heute in der Schweiz zu relevanten Mikroverunreinigungen in Gewässern führen.

Da die Emissionsszenarien oft unvollständig sind, braucht es für eine verlässliche Umwelt-Risikobewertung der Biozide experimentelle Studien. Diese sollten geeignet sein die zulassungsrelevanten Szenarien der BPRL (Environmental Emission Szenarios) auf deren Aussagegehalt und Limitierung zu prüfen. Für viele Produktarten fehlen diese Grundlageninformationen - sogar nach Aussage von Herstellern. Derzeit können für bestimmte Produktarten fast ausschließlich einfache Laborstudien herangezogen werden, da das geläufige Umweltmonitoring bestimmte Wirkstoffe nicht oder nur punktuell-zeitweilig erfasst. Die Bewertung der Biozide beruht daher vor allem auf den Daten der Hersteller und dem „ExpertenWissen“ der Zulassungsbehörden. Eine so schwache Datengrundlage wäre beispielsweise für Pflanzenschutzmittel heute nicht mehr akzeptabel. Basierend auf bestimmten „Mustern“ oder „Klassen“ könnten die Biozide vorgruppiert werden, so dass beispielsweise nur für die persistenteren Wirkstoffe vertiefte Daten vorgelegt werden müssen - dann aber breit und aussagekräftig. In der Studie von Burkhardt et al. (Teil III dieser Beitragsserie) wird gezeigt, in welche Richtung experimentelle Studien und Bewertungen entwickelt werden sollten. Für einzelne prioritäre Wirkstoffe, welche in Fassaden eingesetzt werden, wurde eine expositions- und effektbasierte Bewertung exemplarisch vorgenommen.

\section{Literatur}

Kretschmar E, Müller-Knoche S, Schwarz-Schulz B, Nöh I (2009) Erfahrungen des Umweltbundesamtes mit der EG-BiozidprodukteRichtlinie. Umweltwiss Schadst Forsch, DOI: 10.1007/s12302008-0036-y

Richtlinie 98/8/EG des Europäischen Parlaments und des Rates vom 16. Februar 1998 über das Inverkehrbringen von Biozid-Produkten, Amtsblatt L 123 vom 24.4. 1998

Gesetz zur Umsetzung der Richtlinie 98/8/EG des Europäischen Parlaments und des Rates vom 16. Februar 1998 über das Inverkehrbringen von Biozid-Produkten (Biozid-Gesetz) vom 20. Juni 2002, Bundesgesetzblatt Jahrgang 2002, Teil I, Nr. 40, S. 2076

Schweizerischer Bundesrat, Verordnung vom 18. Mai 2005 über das Inverkehrbringen von und den Umgang mit Biozidprodukten (Biozidprodukteverordnung, VBP)

Schweizerische Biozidprodukteverordnung (VBP): http://www.ad$\min . \mathrm{ch} / \mathrm{ch} / \mathrm{d} / \mathrm{sr} / \mathrm{c} 813 \_12 . \mathrm{html}$

EU Biozidprodukte-Richtlinie (BPRL): http://www.biozide.at/biozidregelungen/eu-richtlinie/;http://ec.europa.eu/environment/biocides/

European Chemical Bureau, Joint Research Centre: http://ecb.jrc. ec.europa.eu/biocides/

Bundesanstalt für Arbeitsschutz und Arbeitsmedizin: http://www. baua.de/de/Chemikaliengesetz-Biozidverfahren/Chemikaliengesetz-Biozidverfahren.html 